\title{
Effect of Processed Honey and Royal Jelly on Cancer-Related Fatigue: A Double-Blind Randomized Clinical Trial
}

\begin{abstract}
Bahram Mofid ${ }^{1}$, Hossein Rezaeizadeh ${ }^{2}$, Abdulkarim Termos ${ }^{3}$, Afshin Rakhsha ${ }^{4}$, Ahmad Rezazadeh Mafi $^{5}$, Taiebeh Taheripanah ${ }^{6}$, Mehran Mirabzadeh Ardakani ${ }^{7}$, Seyed Mohammad Esmaeil Taghavi ${ }^{8}$, Seyyed Alireza Moravveji ${ }^{9}$, Amir Shahram Yousefi Kashi ${ }^{10}$
\end{abstract}

\footnotetext{
${ }^{1}$ Associate Professor, Shohada-e-Tajrish Hospital, Department of Radiation Oncology, Faculty of Medicine, Shahid Beheshti University of Medical Sciences, Tehran, Iran

${ }^{2}$ M.D, Ph.D. of Traditional Persian Medicine, Assistant Professor, Department of Traditional Persian Medicine, School of Traditional Medicine, Tehran University of Medical Science, Tehran, Iran

${ }^{3}$ M.D, Radiation Oncologist, Shohada-e-Tajrish Hospital, Department of Radiation Oncology, Shahid Beheshti University of Medical Sciences, Tehran, Iran

${ }^{4}$ Associate Professor, Shohada-e-Tajrish Hospital, Department of Radiation Oncology, Faculty of Medicine, Shahid Beheshti University of Medical Sciences, Tehran, Iran

${ }^{5}$ M.D, M.Sc., Radiation Oncologist, Shohada-e-Tajrish Hospital, Department of Radiation Oncology, Shahid Beheshti University of Medical Sciences, Tehran, Iran

${ }^{6}$ M.D, Radiation Oncologist, Aja University of Medical Sciences, Tehran, Iran

${ }^{7}$ Pharm. D, Ph.D. of Traditional Persian Medicine, Assistant Professor, Department of Traditional Persian Medicine, School of Traditional Medicine, Tehran University of Medical Science, Tehran, Iran

${ }^{8}$ M.D, Occupational and Environmental Medicine Specialist, Tehran University of Medical Sciences, Tehran, Iran

${ }^{9}$ M.D, M.P.H, Associate Professor, Department of Community Medicine, Kashan University of Medical Sciences, Kashan, Iran

${ }^{10}$ Associate Professor, Shohada-e-Tajrish Hospital, Department of Radiation Oncology, Faculty of Medicine, Shahid Beheshti University of Medical Sciences, Tehran, Iran
}

\section{Type of article: Original}

\begin{abstract}
Background: Cancer-related fatigue (CRF) is experienced by $50 \%$ to $90 \%$ of cancer patients and can severely affect their quality of life and functional capacity. Several randomized trials have recommended various ways to alleviate the symptoms of CRF with or without recourse to medications.

Objective: The aim of this study is to evaluate the effectiveness of processed honey and royal jelly on the symptoms of CRF in cancer patients who are undergoing hormone therapy, chemotherapy, chemo-radiation, or radiotherapy.

Methods: Fifty-two participants from the patients who visited the oncology clinic of Shohada-e-Tajrish hospital in Tehran (Iran) between May 2013 and August 2014 were selected and divided into two groups. The study group (26 patients) received processed honey and royal jelly, while the control group received pure honey. Both groups were instructed to consume their $5 \mathrm{~mL}$ supplement twice daily for 4 weeks. Both groups were assessed at the beginning of the study, after 2 weeks, and then at the end of 4 weeks of treatment. Fatigue was measured using a visual analogue fatigue scale (VAFS) and fatigue severity scale (FSS). The results were compared between the two arms of study, and equality of probability distributions was assessed using a Kolmogorov-Smirnov test.

Results: The mean age of the 52 patients was 54.84. After two and four weeks of treatment with processed honey and royal jelly, VAFS and FSS due to treatment was better in the study group than in the control group, and the
\end{abstract}

\section{Corresponding author:}

Associate Professor Dr. Amir Shahram Yousefi Kashi, Shohada-e-Tajrish Hospital, Department of Radiation Oncology, Faculty of Medicine, Shahid Beheshti University of Medical Sciences, Tehran, Iran.

Tel: +98.2122739200, Fax:+98.2122739200, Email: shahpoo2002@yahoo.com

Received: August 18, 2015, Accepted: February 27, 2016, Published: June 2016

iThenticate screening: September 09, 2015, English editing: May 19, 2016, Quality control: May 20, 2016

(C) 2016 The Authors. This is an open access article under the terms of the Creative Commons Attribution-NonCommercialNoDerivs License, which permits use and distribution in any medium, provided the original work is properly cited, the use is non-commercial and no modifications or adaptations are made. 
differences were statistically significant $(\mathrm{p}<0.001, \mathrm{p}<0.001$, respectively).

Conclusion: To the best of our knowledge, our study provided support for the use of processed honey and royal jelly to ameliorate CRF. The positive results of this study warrant further studies in this field.

Clinical Trial Registration: The study was registered in the Iranian Clinical Trial Registry Center (http://www.irct.ir) with the registration code: IRCT2015081423426N1.

Funding: The authors received no financial support for the research, authorship, and/or publication of this article. Keywords: Fatigue, Cancer, Royal jelly

\section{Introduction}

Cancer-related fatigue (CRF) is a common and treatable symptom, reported in $50 \%-90 \%$ of patients. It can severely affect numerous aspects of the cancer patient's quality of life and functional capacity $(1,2)$. Many cancer patients complain of fatigue as one of the most challenging problems related to cancer (3). Early diagnosis and management of this stressful symptom is of great importance; it is often unreported and consequently does not receive adequate treatment (3). As a result, many patients report that CRF has had profound emotional, mental, physical, and social effects on the quality of their lives. CRF is rarely an isolated symptom and most commonly occurs with other symptoms such as pain, distress, anemia, and sleep disturbances, and it should be diagnosed only after ruling out all other reasons for fatigue, such as treatment complications and especially cancer progression as well as treatable conditions such as hypothyroidism, anemia, and depression (4-6). As patients often experience multiple symptoms that may vary according to diagnosis, treatment, and stage of disease, and because of the significant effects of psychosocial and cultural differences on expressing symptoms by different individuals, exact prevalence of CRF among cancer patients is difficult to determine. However, based on various studies, it is estimated that $60 \%-96 \%$ of cancer patients in treatment experience fatigue, including $60 \%-93 \%$ of those on radiotherapy and $80 \%-96 \%$ of those on chemotherapy (including immune-modulators such as interleukin and interferon) (5-12). For many cancer survivors, fatigue can persist for 5 to 10 years after treatment $(2,3)$. The pathophysiology of CRF has not been adequately elucidated. Anemia, depression, anxiety, immobility, chemotherapy, radiation therapy, and the cancer itself have been shown to contribute to CRF (13). Management of CRF should start with determination of the causative mechanisms of fatigue in oncology patients and addressing the treatable issues such as hypothyroidism, anemia, and depression. Due to the absence of a clear etiology for CRF, combined treatment to reduce symptoms of physical and mental fatigue for cognitive impairment patients should be used (14). It must be mentioned that the treatment should be initiated early to prevent CRF from turning into a chronic problem. Several randomized trials have recommended various ways to alleviate the symptoms of CRF with or without recourse to medications. Nonpharmacological methods usually include psychosocial interventions (such as cognitive behavioral therapeutic approaches) and physical exercise. Several pharmacologic interventions (mainly psychiatric medications) have been recommended for treating CRF; however, there is still no robust data to support the routine use of such medications for this disorder (12-14). Several other studies have evaluated the effects of various food supplements and herbal medication on CRF. Royal jelly is a honey bee secretion that is used in the nutrition of larvae as well as adult queens. The overall material is composed of water $(67 \%)$, crude protein (including small amounts of many different amino acids), monosaccharides, and a relatively high amount of fatty acids. It also contains many trace minerals, some enzymes and antibacterial and antibiotic components but is void of fat-soluble vitamins, A, D, E and $\mathrm{K}$. Several studies so far have shown antioxidant (15-18), immune-modulatory (19-22), and anti-tumoral and antiinflammatory (23-25) characteristics of the royal jelly. In this randomized double-blind placebo-controlled clinical trial, we have assessed the effects of processed honey and royal jelly on the symptoms of CRF.

\section{Material and methods \\ 2.1. Trial design}

Processed honey and royal jelly are made under standard-state conditions with the permission of the Food and Drug Organization of Ministry of Health and Medical Education of Iran. Several unpublished data from dissertations or phase I or II trials with a small number of cases have shown its effectiveness in ameliorating the symptoms of CRF. We conducted this single-center double-blind randomized study after obtaining approval from our local ethics board and after receiving registry codes from the Iranian Registry of Clinical Trials (Registration code: IRCT2015081423426N1). Enrollment of subjects took place between May 2013 and August 2014 at Shohada-eTajrish Hospital in Tehran (Iran). In total, 52 patients were randomized into two groups: 26 participants to the drug group and 26 participants to the placebo group. Demographic data (age and gender), type of malignancy, and its stage (based on TNM staging), type, and duration of treatment were recorded for each participant. Quality of life (performance status) was measured using The Eastern Cooperative Oncology Group (ECOG) score. Fatigue was 
measured using a visual analogue fatigue scale (VAFS) and fatigue severity scale, which is described elsewhere. The primary outcome resulted in changes in the fatigue severity scales and VAFS.

\subsection{Participants}

Inclusion criteria were as follows: age more than 18, having the diagnosis of a solid (nonhematologic) malignancy, visual analogue scale (VAS) more than 4, Karnofsky performance status (KPS) more than 70, serum hemoglobin level of more than $8 \mathrm{~g} / \mathrm{dL}$ with no abrupt decrease (more than $2 \mathrm{~g} / \mathrm{dl}$ ) during the last month, liver enzymes not more than three times above the normal limits, serum total bilirubin less than $2 \mathrm{mg} / \mathrm{dL}$, serum creatinine less than $2 \mathrm{mg} / \mathrm{dL}$, and creatinine clearance more than $60 \mathrm{ml} / \mathrm{mi}$. Having one of these conditions, patients who had started chemotherapy between 6 weeks to 6 months before the start of the study and were supposed to continue chemotherapy for at least 3 more weeks; patients who had started hormone therapy at least 3 months before the start of the study and were supposed to continue the treatment throughout the study period; patients under radiation therapy who were complaining of fatigue; patients whose treatment had ended but still were complaining of fatigue; patients with hypersensitivity to honey, uncontrolled cardiac or respiratory problems, any other serious medical condition, including severe infection or uncontrolled pain, pregnancy, major depression, taking medications with potential effects on fatigue, such as psychiatric medication (anti-depressants, psychostimulants, etc.) or growth factors such as erythropoietin, were excluded from the study.

\subsection{Interventions}

Processed honey and royal jelly are homogeneous substances with the consistency of a fairly fluid paste. They are yellowish in color with brown tinges, have a slightly sour flavor, and are partially soluble in water. Analysis of the characteristics and composition of processed honey and royal jelly was done in Kimiafaam (http://en.kimiafaam.com/bee.php), which is the reference laboratory of the ministry of health. The final product was controlled based on the amount of 10-hydroxydecanoic acids by high-performance liquid chromatography (HPLC) method at two reference laboratories: Kimia-Novin-Azma and Pharmacognosy departments of Tehran University of Medical Sciences. The physicochemical characteristics of processed honey and royal jelly are shown in Table 1 . The study group (26 patients) received processed honey and royal jelly, while the control group received pure honey (without royal jelly). Both groups were instructed to consume their supplement of $5 \mathrm{~mL}$ twice daily for 4 weeks. Both groups were assessed at the beginning of study, after 2 weeks, and then at the end of 4 weeks of treatment.

Table 1. Composition of Processed Honey and Royal Jelly

\begin{tabular}{|l|l|}
\hline Acidity & $17.7 \mathrm{meq} / \mathrm{kg}$ \\
\hline Total ash content & $0.06 \%$ \\
\hline Lipids & $0.04 \%$ \\
\hline Proteins & $0.62 \%$ \\
\hline Sugars & $76.5 \%$ \\
\hline Sucrose & $4.6 \%$ \\
\hline Glucose & $23.63 \%$ \\
\hline Fructose & $47.98 \%$ \\
\hline
\end{tabular}

\subsection{Outcome}

All patients were evaluated before treatment, on the second week, fourth week, and after intervention. During each visit, fatigue was measured using a VAFS and fatigue severity scale. The primary outcome resulted in changes in the fatigue severity scale and VAFS.

\subsection{Sampling}

This study was performed on 52 patients with histologically proven breast cancer, stomach cancer, esophagus cancer, colon cancer, rectal cancer or prostate cancer, who were treated with hormone therapy, chemotherapy, chemo-radiation, or radiotherapy and able to declare their severity of fatigue and were included in the study. These patients took either processed honey and royal jelly (study group) or pure honey (placebo group) after considering the inclusion and exclusion criteria.

\subsection{Randomization}

Fifty-two patients were randomized into two groups, and randomization was accomplished by computer using dynamic allocation with an established algorithm that balances the marginal distribution. The patients were allocated 
randomly into the two groups: 26 participants to processed honey and royal jelly and 26 participants to pure honey group of the same shape. After treatment and randomization, the patients received either processed honey and royal jelly (study group) or pure honey (control group) of the same shape. The patients and physicians were not aware of the kind of medications that had been selected for them.

\subsection{Blinding}

This was a double-blinded study because the kind of medications used for the patients and physicians was blinded to the allotted treatment, and the analysis was based on the treatment received. We tried to diminish the bias by blinding the patients, staff, and physicians of the clinical outcomes data to the type of treatment that was used for each group.

\subsection{Statistical methods}

Both groups were assessed at the beginning of the study, after 2 weeks, and then at the end of 4 weeks of treatment. At each visit, fatigue was measured using a visual analogue fatigue scale (VAFS) and fatigue severity scale. Changes in the VAFS and fatigue severity scales between baseline and each follow-up visit were documented. The results were compared between the two arms of study, those with processed honey and royal jelly and those with pure honey. Equality of probability distributions was assessed using the Kolmogorov-Smirnov test. Wilcoxon and McNemar's statistical tests were used to analyze the data.

\subsection{Research ethics}

All 52 patients signed informed consent. The ethical regulations dictated in the act provided by the research center of Shohada-e-Tajrish Hospital in Shahid Beheshti University of Medical Sciences (reference number of research ethics committee: M/269) were strictly observed. The data are preserved regardless of the patient's names.

\section{Results}

The study included 52 patients (21 males [40.4\%] and 31 females [59.6\%\}). In the study group, nine patients (34.6\%) were male and 17 patients $(65.4 \%)$ were female. In the control group, 12 patients $(46.2 \%)$ were male and 14 patients $(53.8 \%)$ were female. The mean age of total patients was 54.84 years, and the mean age of study and control groups were 55.42 and 54.27 years, respectively. The study group included 14 patients $(53.8 \%)$, and the control group included 13 patients $(50 \%)$ that had breast cancer. Two patients $(7.7 \%)$ in the study group and no patients $(0 \%)$ in the control group had stomach cancer. In the study group, three patients $(11.5 \%)$ and in the control group one patient $(3.8 \%)$ had esophagus cancer. In the study group, no patients $(0 \%)$ and in the control group three patients $(11.5 \%)$ had colon cancer. One patient $(3.8 \%)$ in the study group and no patients $(0 \%)$ in the control group had rectal cancer. Six patients $(23.1 \%)$ in the study group and nine patients $(34.6 \%)$ in the control group were affected by prostate cancer. In the study group, 12 patients $(46.2 \%)$ and in the control group 14 patients $(53.8 \%)$ received hormonal therapy. Two patients $(7.7 \%)$ in the study group and four patients $(15.4 \%)$ in the control group were treated with chemotherapy. In the study group, five patients (19.2\%) and in the control group one patient (3.8\%) received chemo-radiation. Seven patients $(26.9 \%)$ in the study group and seven patients $(26.9 \%)$ in the control group were treated with radiotherapy. There was no statistically significant difference between the two groups in terms of mean age, type of cancer, and type of treatment. The patient and treatment characteristics are summarized in Table 2. There were not any differences between the study and control groups before treatment as FSS, ECOG PS, and VPSS ( $\mathrm{p}=0.541, \mathrm{p}=1, \mathrm{p}=1$, respectively). After two weeks of treatment, the mean FSS was 5.31 with SD of 1.258 in the study group, and it was 7.46 with SD of 1.067 in control group with statistically significant difference $(p<0.001)$. This difference was more prominent after the fourth week of intervention $(\mathrm{p}<0.001)$. After 2 weeks of treatment, the mean ECOG PS was 3.69 with SD of 0.884 in study group, and it was 5.5 with SD of 0.906 in control group with statistically significant difference ( $\mathrm{p}<0.001)$. After 4 weeks, ECOG PS due to treatment was better in the case group compared with that of the control group with statistically significant difference $(p<0.001)$. After 2 weeks, the mean VPSS was 1.69 with SD of 0.618 in patients were treated with honey and royal jelly, and it was 2.46 with SD of 0.508 in patients treated with pure honey with statistically significant difference $(p<0.001)$. This difference was more prominent after the fourth week of intervention $(\mathrm{p}<0.001)$. Comparing the basal fatigue severity scale with the scales measured at 2 and 4 weeks after the intervention showed that the participants in the study group did have lower fatigue scores, and this difference was more prominent after the fourth week of intervention (Table 3). 
Table 2. Patient and Treatment Characteristics of the Patients in the Two Groups

\begin{tabular}{|l|l|l|l|l|}
\hline Characteristics & & Study & Control & Total \\
\hline Mean Age (years) & & 55.42 & 54.27 & 54.84 \\
\hline Gender & Male & $9(34.6 \%)$ & $12(46.2 \%)$ & $21(40.4 \%)$ \\
\cline { 2 - 5 } & Female & $17(65.4 \%)$ & $14(53.8 \%)$ & $31(59.6 \%)$ \\
\cline { 2 - 5 } & Total & $26(100 \%)$ & $26(100 \%)$ & $52(100 \%)$ \\
\hline \multirow{5}{*}{ Type of Cancer } & Breast & $14(53.8 \%)$ & $13(50.0 \%)$ & $27(51.9 \%)$ \\
\cline { 2 - 5 } & Stomach & $2(7.7 \%)$ & $0(0 \%)$ & $2(3.8 \%)$ \\
\cline { 2 - 5 } & Esophagus & $3(11.5 \%)$ & $1(3.8 \%)$ & $4(7.7 \%)$ \\
\cline { 2 - 5 } & Colon & $0(0 \%)$ & $3(11.5 \%)$ & $3(5.8 \%)$ \\
\cline { 2 - 5 } & Rectum & $1(3.8 \%)$ & $0(0 \%)$ & $1(1.9 \%)$ \\
\cline { 2 - 5 } & Prostate & $6(23.1 \%)$ & $9(34.6 \%)$ & $15(28.8 \%)$ \\
\cline { 2 - 5 } & Total & $26(100.0 \%)$ & $26(100.0 \%)$ & $52(100.0 \%)$ \\
\hline Type of Treatment & Hormone therapy & $12(46.2 \%)$ & $14(53.8 \%)$ & $26(50.0 \%)$ \\
\cline { 2 - 5 } & Chemotherapy & $2(7.7 \%)$ & $4(15.4 \%)$ & $6(11.5 \%)$ \\
\cline { 2 - 5 } & Chemoradiation & $5(19.2 \%)$ & $1(3.8 \%)$ & $6(11.5 \%)$ \\
\cline { 2 - 5 } & Radiotherapy & $7(26.9 \%)$ & $7(26.9 \%)$ & $14(26.9 \%)$ \\
\cline { 2 - 5 } & Total & $26(100.0 \%)$ & $26(100.0 \%)$ & $52(100.0 \%)$ \\
\hline
\end{tabular}

Table 3. Comparison of the Fatigue and Performance Status at the Beginning (Base) After the Second and Fourth Weeks of Study

\begin{tabular}{|c|c|c|c|c|}
\hline \multirow{2}{*}{\multicolumn{2}{|c|}{ Characteristics }} & Study & Control & \multirow{2}{*}{ p-value } \\
\hline & & Mean $\pm{S D^{a}}^{a}$ & Mean \pm SD & \\
\hline \multirow[t]{3}{*}{$\mathrm{FSS}^{\mathrm{b}}$} & FSS base & $8.08 \pm 0.628$ & $7.96 \pm 0.720$ & 0.541 \\
\hline & FSS $2^{\text {nd }}$ week & $5.31 \pm 1.258$ & $7.46 \pm 1.067$ & $<0.001$ \\
\hline & FSS $4^{\text {th }}$ week & $2.77 \pm 1.583$ & $6.58 \pm 1.724$ & $<0.001$ \\
\hline \multirow[t]{3}{*}{ ECOG PS } & ECOG base & $6.00 \pm 0.693$ & $6.00 \pm 0.566$ & 1 \\
\hline & ECOG $2^{\text {nd }}$ week & $3.69 \pm 0.884$ & $5.50 \pm 0.906$ & $<0.001$ \\
\hline & ECOG $4^{\text {th }}$ week & $1.96 \pm 0.999$ & $4.88 \pm 1.306$ & $<0.001$ \\
\hline \multirow[t]{3}{*}{ VAFS $^{d}$} & VAFS base & $2.69 \pm 0.471$ & $2.69 \pm 0.549$ & 1 \\
\hline & VAFS $2^{\text {nd }}$ week & $1.69 \pm 0.618$ & $2.46 \pm 0.508$ & $<0.001$ \\
\hline & VAFS $4^{\text {th }}$ week & $0.65 \pm 0.846$ & $2.15 \pm 0.784$ & $<0.001$ \\
\hline
\end{tabular}

aSD: standard deviation; ${ }^{\mathrm{b}} \mathrm{FSS}$ : fatigue severity scale; ${ }^{\mathrm{c}} \mathrm{ECOG}$ PS: Eastern Cooperative Oncology Group Performance Status score; ${ }^{\mathrm{d}}$ VAFS: visual analogue fatigue scale.

\section{Discussion}

The exact reason for cancer fatigue is unknown. CRF is multifactorial and may be related to both the disease process and treatments, including surgery, chemotherapy, and radiation therapy. Several etiology factors for the progression of CRF have been suggested, but there is no lab test for identifying CRF as objective method (13). Therefore, a wide range of causes of physical, emotional, cognitive, and psychological often cannot easily be separated from the development of CRF helps. Some of the proposed mechanism include cytokine dysregulation, changes in serotoninergic CNS, a disorder of circadian secretion of melatonin, and the sleep-wake rhythm, and polymorphism gene regulatory proteins of oxidative phosphorylation $(13,16,17)$. Besides, numerous other factors can contribute to the severity of CRF, such as poor nutrition, anemia, hypothyroidism, medications for other illnesses, pain, stress, and depression. Some of the medications whose safety and efficacy have been studied in randomized controlled trials include hematopoietic growth factors, psychostimulants (such as methylphenidate and modafinil), corticosteroids, Donepezil, multivitamins, thyrotropin-releasing hormone (TRH), and phytotherapeutic agents (Ginseng) $(14,15)$. The study by Moraska et al. on 148 patients with at least one month history of CRF, methylphenidate, as compared with placebo, failed to improve CRF. A subset analysis, however, suggested that patients with more severe fatigue and/or with more advanced disease (e.g., in patients with stage III or IV disease) did have some fatigue improvement with methylphenidate $(26,27)$. Effects of antidepressants on alleviating the symptoms of CRF have not been promising either. Several studies have shown that medications such as paroxetine and sertraline-although able to reduce the symptoms of depression-have no influence on fatigue in patients receiving chemotherapy. A possible explanation could be that CRF does not involve a reduction in brain 5-HT levels (28-30). Several studies also have evaluated the effects of various food supplements and herbal medication of CRF. 
Coenzyme Q 10, L-Carnitine and guarana have been evaluated in placebo-controlled trials for CRF; however, they have been found to be no more helpful than placebos (31). In contrast, some studies on supplements and herbal medication have shown promising results. Barton et al., in their study on 364 patients, showed that consumption of American ginseng, $2000 \mathrm{mg}$ daily over an 8-week period, can be beneficial in alleviating the symptoms of CRF (31). A study on the effects of Bojungikki-tang (a widely used herbal prescription in traditional medicine in China, Japan, and Korea) on 40 patients has also shown promising results in managing the symptoms and improving quality of life in patients with CRF (32). Several studies so far have shown antioxidant (15-18) and immune-modulatory (19-22) characteristics of the royal jelly. Therefore, it seems that it might be effective in improvement of the symptoms of CRF. Some studies have even shown anti-tumoral and anti-inflammatory properties of royal jelly (23-25). In this study, a processed form of royal jelly proved to be beneficial in the management of CRF. Kamakura et al. investigated the antifatigue effect of royal jelly in male mice and reported that royal jelly can ameliorate physical fatigue after exercise (33). Fauzi et al. investigated the anticancer potential of Tualang honey (Agromas) in human breast and cervical cancer cell lines as well as in the normal breast epithelial cell line. Their study showed that Tualang honey had significant anticancer activity against human breast and cervical cancer cell lines (34). Another study on 30 patients showed that the use of Life-Mel Honey in patients who are at high risk of developing neutropenia as a result of chemotherapy decreases the risk of pancytopenia and the need for colony-stimulating factors (35). The Erem et al. study showed that royal jelly may be effective as an immune-modulatory agent in Graves' disease (36). Another study demonstrated that 10-Hydroxy-2-decenoic acid from royal jelly may be of potential therapeutic value in inhibiting joint destruction in rheumatoid arthritis (37). Our study also showed that royal jelly can be considered as an effective treatment modality to manage CRF. Royal jelly has no known adverse effects, and, following the recommendation, the patients' compliance was excellent, as it was considered as a food supplement, not a drug. Our study faced two limitations. The first was the number of cases. Although we recruited more participants than needed, and the number of our cases was more than that of many similar studies, still the number of cases was quite small. The second limitation was the short duration of intervention. Trials with a larger number of patients and longer duration of intervention can further clarify the role of processed honey and royal jelly in managing $\mathrm{CRF}$.

\section{Conclusions}

In this uni-centric, double-blind, randomized clinical trial, it was shown that processed honey and royal jelly had a strong effect to ameliorate CRF. While modern premedication or postmedication drugs such as hematopoietic growth factors, methylphenidate, and donepezil can reduce CRF, the application of traditional processed honey and royal jelly treatments to ameliorate $\mathrm{CRF}$ requires an intentional transfer of historical concepts to modern treatment drugs. However, more research is necessary to understand its exact role in this regard.

\section{Acknowledgments:}

This article has been extracted from the thesis written by Mr Abdulkarim Termos in School of Medicine, Shahid Beheshti University of Medical Sciences (Registration No: 269). The authors would like to thank the residents of radiation oncology and staff of the Radiotherapy Department at Shohada-e-Tajrish Hospital for their contributions to the treatment and maintenance of our patients' records.

Clinical Trial Registration: The study was registered in the Iranian Clinical Trial Registry Center (http://www.irct.ir) with the registration code: IRCT2015081423426N1.

Funding: The authors received no financial support for the research, authorship, and/or publication of this article.

\section{Conflict of Interest:}

There is no conflict of interest to be declared.

\section{Authors' contributions:}

All authors contributed to this project and article equally. All authors read and approved the final manuscript.

\section{References:}

1) Gupta $D$, Lis CG, GrutschJF. The relationship between cancer-related fatigueand patient satisfaction with quality of life in cancer. J Pain Symptom Manage. 2007; 34(1):40-7. Doi: 10.1016/j.jpainsymman.2006.10.012. PMID: 175321791 
2) Mock V, Atkinson A, Barsevick AM, Berger AM, Cimprich B, Eisenberger MA, et al. Cancer-related fatigue. Clinical Practice Guidelines in Oncology. J NatlComprCancNetw. 2007; 5(10):1054-78.Review. PMID: 18053429

3) Stone P, Richardson A, Ream E, Smith AG, Kerr DJ, Kearney N. Cancer-related fatigue: inevitable, unimportant and untreatable? Results of a multi-centre patient survey. Cancer Fatigue Forum. Ann Oncol. 2000; 11(8):971-5. Doi: 10.1023/A: 1008318932641. PMID: 11038033

4) Atkins MB, MierJW, Parkinson DR, Gould JA, BerkmanEM, Kaplan MM. Hypothyroidism after treatment with interleukin-2 and lymphokine-activated killer cells. N Engl J Med. 1988; 318(24):1557- 63. Doi: 10.1056/NEJM198806163182401. PMID: 3259674

5) Jereczek-Fossa BA, Santoro L, Alterio D, Franchi B, Fiore MR, Fossati P et al. Fatigue during head-andneck radiotherapy: prospective study on 117 consecutive patients. Int J RadiatOncolBiol Phys. 2007; 68(2):403-15. Doi: 10.1016/j.ijrobp.2007.01.024

6) LittlewoodTJ, Kallich JD, San Miguel J, Hendricks L, Hedenus M. Efficacy of darbepoetinalfa in alleviating fatigue and the effect of fatigue on quality of life in anemic patients with lymphoproliferative malignancies. J Pain Symptom Manage. 2006; 31(4):317-25. Doi: 10.1016/j.jpainsymman.2005.08.013. PMID: 16632079

7) Lis CG, Rodeghier M, GrutschJF, Gupta D. Distribution and determinants of patient satisfaction in oncology with a focus on health related quality of life. BMC Health Serv Res. 2009; 9: 19. Doi: 10.1186/1472-6963-9-190. PMID: 19845942. PMCID: PMC2770467

8) Curt GA, Breitbart W, Cella D, Groopman JE HorningSJ, Itri LM, et al. Impact of cancer-related fatigue on the lives of patients: new findings from the Fatigue Coalition. Oncologist 2000; 5(5):353-60. Doi: 10.1634/theoncologist.5-5-353. PMID: 11040270

9) VogelzangNJ, Breitbart W, Cella D, Curt GA, Groopman JE, HorningSJ, et al. Patient, caregiver, and oncologist perceptions of cancer-related fatigue: results of a tripart assessment survey. The Fatigue Coalition. SeminHematol. 1997; 34(3 Suppl 2):4-12. PMID: 9253778

10) Jereczek-Fossa BA, MarsigliaHR, Orecchia R. Radiotherapy-related fatigue. Crit Rev OncolHematol. 2002; 41(3):317-25. Review. Doi: 10.1016/S1040-8428(01)00143-3

11) Malik UR, Makower DF, Wadler S. Interferon-mediated fatigue. Cancer. 2001 Sep 15; 92 (6 Suppl):1664-8. Doi: 10.1002/1097-0142(20010915)92:6+<1664: AID-CNCR1494>3.0.CO;2-9

12) Barton DL, Liu H, Dakhil SR, et al. Wisconsin Ginseng (Panaxquinquefolius) to Improve Cancer-Related Fatigue: A Randomized, Double-Blind Trial, N07C2. JNCI Journal of the National Cancer Institute. 2013; 105(16):1230-8. Doi: 10.1093/jnci/djt181

13) Wang XS. Pathophysiology of cancer-related fatigue.Clin J OncolNurs. 2008 Oct; 12(5 Suppl):11-20. Doi: 10.1188/08.CJON.S2.11-20. PMID: 18842520. PMCID: PMC3281752

14) Horneber M, Fischer I, Dimeo F, RüfferJU, Weis J. Cancer-Related Fatigue: Epidemiology, Pathogenesis, Diagnosis, and Treatment. Deutsches Ärzteblatt International. 2012; 109(9):161-172. PMID: 22461866. PMCID: PMC3314239

15) Azab KS, et al: Royal jelly modulates oxidative stress and tissue injury in gamma irradiated male wister albino rats.N Am J Med Sci. 2011 Jun; 3(6):268-76. Doi: 10.4297/najms.2011.3268. PMID: 22540097. PMCID: PMC3336918

16) Karadeniz A, Simsek N, Karakus E, Yildirim S, Kara A, Can I, et al: Royal JellyModulates Oxidative Stress and Apoptosis in Liver and Kidneys of Rats Treated with Cisplatin. Oxid Med Cell Longev. 2011; 2011: 981793. Doi: 10.1155/2011/981793.

17) Silici S, Ekmekcioglu O, Kanbur M, Deniz K. The protective effect of royal jelly against cisplatin-induced renal oxidative stress in rats. World J Urol. $2011 \mathrm{Feb}$; 29(1):127-32. Doi: 10.1007/s00345-010-0543-5. PMID: 20369241

18) Cemek M, Yılmaz F, Büyükokuroğlu ME, Büyükben A, Aymelek F, Ayaz A. Serum and liver tissue bioelement levels, and antioxidant enzyme activities in carbon tetrachloride-induced hepatotoxicity: protective effects of royal jelly. J Med Food. 2012 Aug; 15(8):747-52. Doi: 10.1089/jmf.2012.0010. PMID: 22510102

19) Othman NH: Honey and Cancer: Sustainable Inverse Relationship Particularly for Developing Nations-A Review. Evidence-Based Complementary and Alternative Medicine. 2012, Article ID: 410406

20) Pourahmad M, Sobhanian S. Effect of Honey on the Common Cold. Archives of Medical Research; 2009, Vol 40, Issue 3, Pages 224-225. Doi: 10.1016/j.arcmed.2009.01.001. PMID: 19427976

21) Al-Waili NS, Al-Waili TN, Al-Waili AN, Saloom KS. Influence of Natural Honey on Biochemical and Hematological Variables in AIDS: A case study. Scientific World Journal. 2006 Feb 2; 6:1985-9. Doi: 10.1100/tsw.2006.331. PMID: 17369997 
22) Oka H, Emori Y, Kobayashi N, Hayashi Y, Nomoto K.Suppression of allergic reactions by royal jelly in association with the restoration of macrophage function and the improvement of Th1/Th2 cell responses. Int Immunopharmacol. 2001 Mar; 1(3):521-32. Doi: 10.1016/S1567-5769(00)00007-2

23) Nakaya M, Onda H, Sasaki K, Yukiyoshi A, Tachibana H, Yamada K. Effects of royal jelly on Bisphenol A-induced proliferation of human breast cancer cells. BiosciBiotechnolBiochem. 2007 Jan; 71(1):253-5. Doi: 10.1271/bbb.60453. PMID: 17213647

24) Townsend GF, Morgan JF, Tolnai S, Hazlett B, Morton HJ, ShuelRW.Studies on the in vitro antitumor activity of fatty acids. I. 10-Hydroxy-2-decenoic acid from royal jelly. Cancer Res. 1960; 20:503-10. PMID: 13839101

25) Tamura T, Fujii A, Kuboyama N. Antitumor effects of royal jelly (RJ).Nihon YakurigakuZasshi. 1987 Feb; 89(2):73-80. Doi: 10.1254/fpj.89.73. PMID: 3570105

26) Campos MP, Hassan BJ, Riechelmann R, Del Giglio A. Cancer-related fatigue: a review. Rev Assoc Med Bras. 2011 Mar-Apr; 57(2):211-9. Doi: 10.1590/S0104-42302011000200021. PMID: 21537710

27) Moraska AR, Sood A, Dakhil SR, Sloan JA, Barton D, Atherton PJ, et al. Phase III, randomized, doubleblind, placebo-controlled study of long-acting methylphenidate for cancer-related fatigue. North Central Cancer Treatment Group NCCTG-N05C7 trial. J ClinOncol 2010; 28:3673. Doi: 10.1200/JCO.2010.28.1444. PMID: 20625123, PMCID: PMC2917307

28) Parker KP, Bliwise DL, Ribeiro M, Jain SR, Vena CI, Kohles-Baker MK et al. Sleep/Wake patterns of individuals with advanced cancer measured by ambulatory polysomnography. J ClinOncol. 2008; 26(15):2464-72. Doi: 10.1200/JCO.2007.12.2135. PMID: 18487566

29) Stockler MR, O'Connell R, Nowak AK, Goldstein D, Turner J, Wilcken NR, et al. Effect of sertraline on symptoms and survival in patients with advanced cancer, but without major depression: a placebocontrolled double-blind randomised trial. Lancet Oncol. 2007; 8(7):603-12. Doi: 10.1016/S14702045(07)70148-1

30) Palesh O, Mustian KM, Roscoe JA, et al. Effect of paroxetine on depression and insomnia in 547 fatigued cancer patients undergoing chemotherapy (abstract). J ClinOncol 2008; 26:502s.

31) Barton DL, SooriGS, Bauer BA, Sloan JA, Johnson PA, Figueras C, et al. Pilot study of Panaxquinquefolius (American ginseng)to improve cancer-related fatigue: a randomized, double-blind, dose-finding evaluation: NCCTG trial N03CA. Support Care Cancer. 2010 Feb; 18(2):179-87. Doi: 10.1007/s00520-009-0642-2. PMID: 19415341. PMCID: PMC3911883

32) JeongJS, RyuBH, Kim JS, Park JW, Choi WC, Yoon SW. Bojungikki-Tang for Cancer-Related Fatigue: A Pilot Randomized Clinical Trial. Integr Cancer Ther. 2010 Dec; 9(4):331-8. Doi: 10.1177/1534735410383170. PMID: 21059621

33) Kamakura M, Mitani N, Fukuda T, Fukushima M. Antifatigue effect of fresh royal jelly in mice. J NutrSciVitaminol (Tokyo). 2001 Dec; 47(6):394-401. Doi: 10.3177/jnsv.47.394

34) Fauzi AN, Norazmi MN, Yaacob NS. Tualang honey induces apoptosis and disrupts the mitochondrial membrane potential of human breast and cervical cancer cell lines. Food ChemToxicol. 2011 Apr; 49(4):871-8. Doi: 10.1016/j.fct.2010.12.010. PMID: 21167897

35) Zidan J, Shetver L, Gershuny A, Abzah A, Tamam S, Stein M, Friedman E.Prevention of ChemotherapyInduced Neutropenia by Special Honey Intake.MedOncol. 2006; 23(4):549-52. Doi: 10.1385/mo:23:4:549

36) Erem C, Deger O, Ovali E, BarlakY.The Effects of Royal Jelly on Autoimmunity in Graves' Disease. Endocrine. 2006; 30(2):175-83. Doi: 10.1385/ENDO:30:2:175

37) Yang XY, Yang DS, Wei-Zhang, Wang JM, Li CY, Hui-Ye, et al. 10-Hydroxy-2-decenoic acid from Royal jelly: A potential medicine for RA. J Ethnopharmacol. 2010 Mar 24; 128(2):314-21. Doi: 10.1016/j.jep.2010.01.055. PMID: 20138211 